\section{Tumorbedingte Knochenschmerzen: mit Pregabalin die Radiotherapie unterstützen?}

\author{
Kann mit einem Wirkstoff, der an einen Kalziumkanal bindet, die Schmerz- \\ übertragung gehemmt und die Wirkung einer Radiotherapie bei metastasen- \\ bedingten Knochenschmerzen verstärkt werden?
}

G oldstandard in der Therapie von tumorbedingten Knochenschmerzen ist die Radiotherapie. Doch nur bei etwa jedem fünften Patienten lassen sich die Schmerzen damit vollständig beseitigen. Tierexperimentelle Befunde, wonach Substanzen wie Pregabalin und Gabapentin die Schmerzübertragung hemmen und so die Wirkung der Strahlentherapie unterstützen könnten, wurden nun in einer randomisierten Studie klinisch getestet.

233 Patienten mit radiologisch nachweisbaren Knochenmetastasen erhielten randomisiert eine Radiotherapie (8 Gy in 1 oder 20 Gy in 5 Fraktionen) plus Pregabalin $(\mathrm{n}=116)$ oder Placebo $(\mathrm{n}=$ 117). 35 Tage lang nahmen die Patienten entweder zweimal täglich $75 \mathrm{mg}$ Pregabalin oder Placebo ein. Am 1., 8., 15. und 22. Tag wurde die Medikation nach einer Einschätzung der Analgesie angepasst. Primärer Endpunkt war das Therapieansprechen, das heißt die Redukti- on der Schmerzen um mindestens zwei Punkte bis Woche 4 bei mindestens stabiler Opioiddosis.

Am häufigsten litten die Studienteilnehmer unter einem Prostata-, Mammaoder Lungenkarzinom. Im PregabalinArm erreichten 45 Patienten $(38,8 \%)$ den primären Endpunkt, im Placeboarm 47 (40,2\%; Odds Ratio 1,07; p = 0,816). Es gab zwischen den Behandlungsarmen keine signifikanten Unter- schiede hinsichtlich Schmerzintensität, Beeinträchtigung von Aktivitäten durch Schmerzen oder Lebensqualität, wohl aber bezüglich Stimmung $(\mathrm{p}=0,031)$ und Durchbruchschmerzen $(p=0,037)$ - jeweils zugunsten der Therapie mit Pregabalin.

Fazit: Die Ergebnisse der Studie sprechen nicht für die Zugabe von Pregabalin zur Radiotherapie bei krebsbedingten Knochenschmerzen. Künftig sollte die Wirkung von Pregabalin bei Knochenschmerzen mit neuropathischer Komponente untersucht werden.

Kathrin von Kieseritzky

Fallon $\mathrm{M}$ et al. Randomized Double-Blind Trial of Pregabalin Versus Placebo in Conjunction With Palliative Radiotherapy for Cancer-Induced Bone Pain. J Clin Oncol. 2016;34(6):550-6.

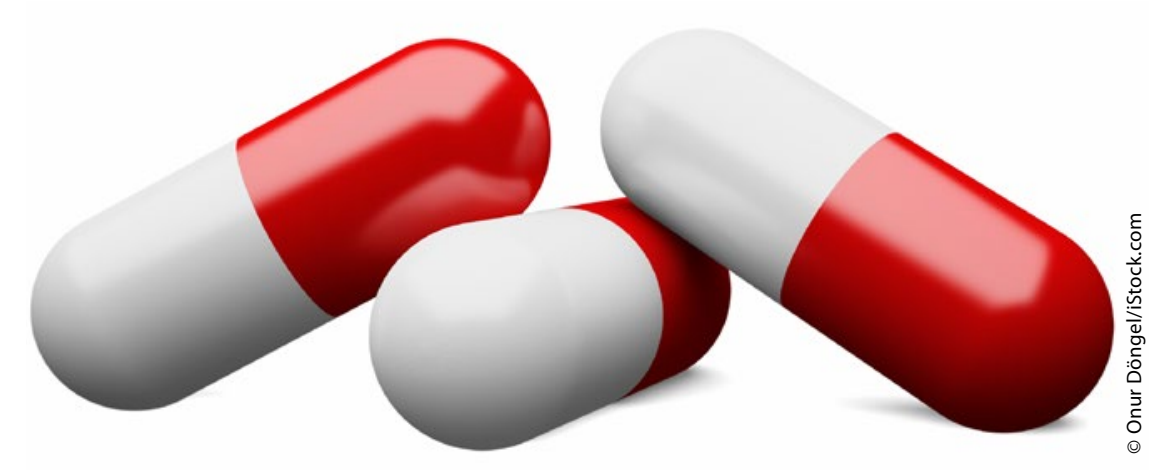

\title{
mCRPC: Auch ältere Männer profitieren von Enzalutamid
}

\section{In Subgruppenanalysen der Studie PREVAIL wurden Wirksamkeit und Sicherheit von Enzalutamid bei Älteren mit mCRPC untersucht.}

P REVAIL und AFFIRM haben gezeigt, dass der orale Androgenrezeptorinhibitor Enzalutamid das Überleben von Männern mit metastasiertem kastrationsresistentem Prostatakarzinom (mCRPC) im Vergleich zu Placebo verlängert, und zwar sowohl vor (PREVAIL) als auch nach Docetaxel-Therapie (AFFIRM). In einer Post-hoc-Analyse von AFFIRM war das Überleben sowohl bei älteren als auch bei jüngeren Patienten verlängert, bei vergleichbarer Verträglichkeit und Sicherheit. In Subgrup- penanalysen von PREVAIL wurden nun Wirksamkeit und Sicherheit von Enzalutamid bei chemotherapienaiven Männern im Alter von $<75(\mathrm{n}=1.108)$ und $\geq$ 75 Jahren $(n=609)$ untersucht. Primäre Endpunkte waren das Gesamtüberleben (OS) und das Überleben ohne radiologische Progression.

Die Älteren hatten zu Studienbeginn einen schlechteren Performancestatus, häufiger kardiovaskuläre Erkrankungen und über 20 Knochenmetastasen sowie einen höheren PSA-Wert. Das OS war bei ihnen unter Enzalutamid signifikant länger als unter Placebo (32,4 vs. 25,1 Monate; Hazard Ratio [HR] 0,61; $\mathrm{p}=0,0001$ ), ebenso das Überleben ohne radiologische Progression (Median nicht erreicht vs. 3,7 Monate; HR 0,17; $\mathrm{p}<0,0001)$. Bei den jüngeren Männern wurden vergleichbare Verbesserungen erreicht. Zudem verbesserte Enzalutamid bei den älteren Männern alle sekundären und exploratorischen Endpunkte.

Fazit: Ältere, chemotherapienaive Männer profitieren von Enzalutamid hinsichtlich des Gesamtüberlebens und Überlebens ohne radiologische Progression. Die Substanz wurde auch von älteren Männern gut vertragen. Judith Neumaier

Graff JN et al. Efficacy and safety of enzalutamide in patients 75 years or older with chemotherapy-naive metastatic castrationresistant prostate cancer: results from PREVAIL. Ann Oncol. 2016;27(2):286-94. 